\title{
Permeability of Fractured Media under Confining Pressure: A Simplified Model
}

\author{
Alberto Roman ${ }^{*, 1}$, Goodarz Ahmadi ${ }^{1}$, Kathleen A. Issen ${ }^{1}$ and Duane H. Smith ${ }^{2}$ \\ ${ }^{1}$ Department of Mechanical and Aeronautical Engineering, Clarkson University, Potsdam, NY 13699-5725, USA; \\ ${ }^{2}$ National Energy Technology Laboratory, U.S. Department of Energy, Morgantown, WV, 26507-0880, USA
}

\begin{abstract}
An elastic-plastic model was developed to describe the deformation and permeability of fractured rocks under confining pressure. Elastic and elastic-plastic contact models were used in the model to analyze the deformations of a representative element containing a single fracture under various confining pressures. The present work utilized elements from contact mechanics and viscous flows to derive an expression for permeability of fractured rocks under a range of confining pressures. The elastic-plastic model predictions for coal permeability under loading and unloading confining pressure were compared with the experimental data. The new model exhibited trends similar to the data for the range of confining pressures used in the experiments. The model can be used to predict the permeability of other fractured media with different material properties.
\end{abstract}

Keywords: asperity, permeability, elastic-plastic contact, hysteresis.

\section{INTRODUCTION}

Carbon dioxide $\left(\mathrm{CO}_{2}\right)$ sequestration in geological reservoirs has emerged as an important practical approach for large scale carbon management. Thus, understanding the nature of flows in fractured rocks and porous media, and the availability of appropriate computational models, are of critical importance for effective design and operation of the geological sequestration process. Pumping $\mathrm{CO}_{2}$ into geological reservoirs is associated with significant changes in the effective pressure and stresses. In particular, the permeability of the reservoir will change. Thus, this paper seeks to model changes in permeability due to changes in effective pressures.

Previous researchers [1] have formulated mathematical models for evaluating the permeability of porous and fractured media, but omitted the effect of confining pressure and the associated deformation. More recently, a few publications have appeared that include the effect of confining and pore pressures on the effective permeability of fractured media (Walsh and Grosebaugh [2]), but were limited to an elastic deformation assumption-that is, they did not account for plastic deformation (unrecoverable strains) in their analyses. Recent experimental data by Smith and Jikich [3] clearly showed the hysteretic effects in the permeability for loading and unloading of the sample, which suggests the presence of plastic deformation.

Actual rock fracture surfaces are rough, resulting in asperity contact when pressure is applied; therefore, a

*Address correspondence to this author at the Department of Mechanical and Aeronautical Engineering, Clarkson University, Potsdam, NY 136995725, USA; Tel: (315)212-3570; E-mails: romana2@clarkson.edu; alberto10453@yahoo.com contact mechanics approach could be useful in modeling fracture closure. A significant amount of work has been conducted to determine the inception of yielding of contact surfaces. For example, Gao et al., [4] and Krithivasan and Jackson [5] employed an elastic-plastic sinusoidal surface in contact with a rigid surface to determine the inception of yielding. Kadin et al., [6] and Etsion et al., [7] developed an elastic-plastic model for a rough surface in contact with a rigid surface to analyze the unloading behavior of the contact surfaces. Jackson et al., [8] carried out finite element analyses to determine residual stresses and deformations in hemispherical contacts. The finite element results revealed a significant amount of permanent deformation of the hemispherical asperities.

The present study focuses on the development of an elastic-plastic model for deformation of fractured rocks under various confining pressures. The main goal is to develop a model for evaluating permeability of the fractured media that includes elastic, as well as plastic, deformation during loading and unloading. Inclusion of the plastic deformation in the model is critical for providing a more accurate description of the permeability of fractured media under varying confining pressures, and in providing a possible explanation for the variation of permeability during loading and unloading that was reported in the experiments [3].

\section{MODEL FORMULATION}

\subsection{Elastic-Plastic Model}

Fig. (1) shows the schematic of the representative element used in the model development. The representative element consists of a rigid impermeable block with an idealized fractured surface. The contact asperities within the fracture are, however, deformable. Under the action of the 
net confining pressure, $P_{c}$, the asperities undergo elastic, as well as plastic, deformation depending on the level of external loading. The permeability was modeled as a function of the net confining pressure, $P_{c}$, effective height of fracture aperture, $h_{o}$, and total height of the representative element, $H_{o}$. The net confining pressure is the difference between the actual confining pressure and the fluid pore pressure. The deformable asperities are assumed to have isotropic material characteristics. The contact points of the asperities were idealized as semi-spherical, with radius of curvature $R$.

The bulk of the matrix is treated as rigid, but the asperities are assumed to deform under the external load. For elastic deformation, the Hertzian contact model [9] was used in the analysis. When the stress at the contact point exceeds certain limits, plastic deformation is assumed to occur and the Maugis-Pollock model [10] is used. The contact models relate the contact area to the elastic and plastic deformations.

The flow through the fracture was idealized by flow between two smooth, parallel plates. The flow rate per unit width in the Poiseuille's flow is given as

$q=-\frac{h^{3}}{12 \mu}\left(\frac{d p}{d x}\right)$,

where $p$ is the fluid pressure and $\mu$ is the fluid viscosity. From Darcy's law, the flow rate per unit width through a porous media is given by

$q=\frac{-k H_{o}}{\mu}\left(\frac{d p}{d x}\right)$,

where $k$ is the permeability and $H_{o}$ is the height of the porous media sample as shown in Fig. (1). Equating Equations (1) and (2), the effective permeability of the fractured media is given as

$k=\frac{h^{3}}{12 H_{o}}$.

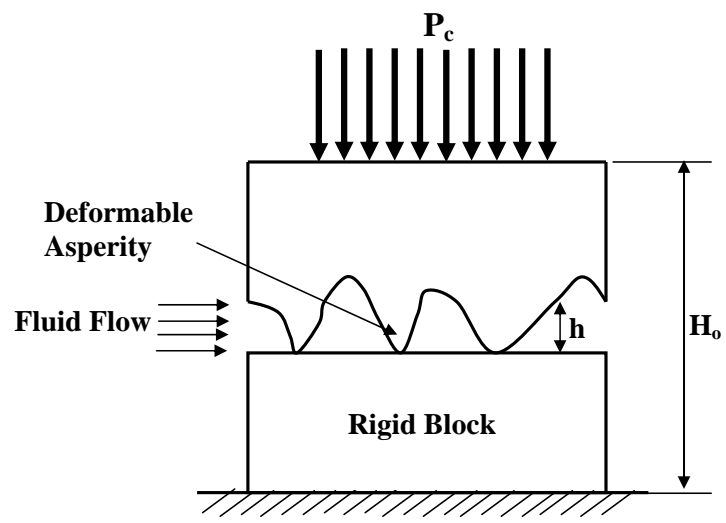

Fig. (1). Schematic of the representative element of fractured media.

Under external pressure, the fractured media (asperities) deforms, and the fracture aperture changes, which leads to the changes in the effective permeability. Thus, the deformation of the asperities under external load needs to be evaluated. Here, it is assumed that there are $n$ uniformly sized asperities per unit area connecting the sides of the fracture. The deformation of the asperities is discussed in the next section.

\subsection{Contact of Rough Surfaces}

Consider an asperity with radius of curvature $R$ in contact with a flat surface. Assume that a force $F$ is exerted on the asperity, which forms a contact area of radius $a$. Fig. (2) shows the schematic of the contact deformation, where $\alpha$ is the penetration distance. From the geometry of the contact, since $a<<R$, it follows that

$\alpha=\frac{a^{2}}{2 R}$

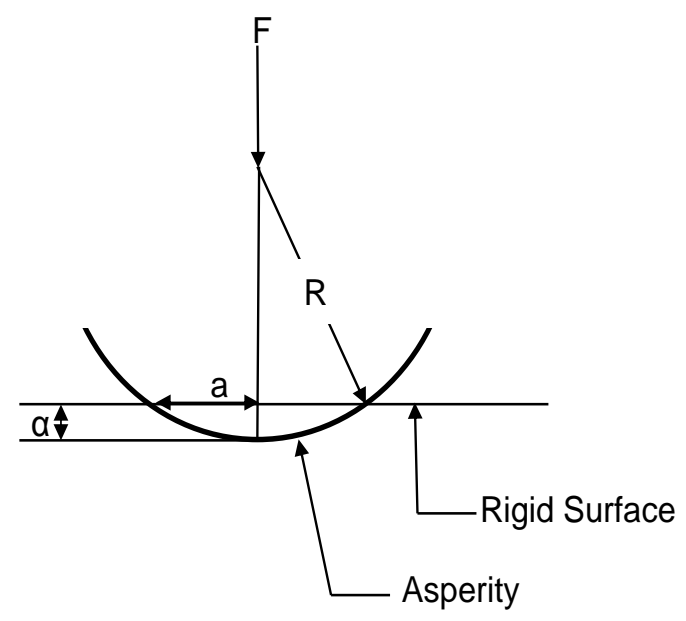

Fig. (2). Schematic for an asperity in contact with a plane surface.

For elastic contact, the classical Hertz model [9] is given as

$a^{3}=\frac{R F}{K}$,

where $F$ is the force exerted on the asperity, and $K$ is the composite modulus given by

$K=\frac{2}{3}\left[\frac{E}{1-v^{2}}\right]$,

where $E$ is the Young's modulus and $v$ is the Poisson's ratio. Here, it is assumed that the material properties of the asperity are isotropic. The isotropy assumption produces reasonable results; however, a more realistic constitutive model could be implemented in future work.

Substituting Equation (5) into Equation (4) provides an expression for the elastic deformation of the asperity tip:

$\alpha_{e}=\frac{1}{2 R}\left[\frac{R P}{K n}\right]^{2 / 3}$,

where $P$ is the external confining pressure, and $n$ is the number of asperities per unit area (note that $F=P / n$ ). The subscript $e$ denotes an elastic effect.

For plastic deformation, the Maugis-Pollock model [10] leads to 
Table 1. Idealized Fracture Model Parameters [9]

\begin{tabular}{|l|c|c|}
\hline \multicolumn{1}{|c|}{ Symbols } & Values & Dimensions \\
\hline \hline Young's Modulus $[E]$ & 4.24 & $\mathrm{MPa}$ \\
\hline Possion's Ratio $[v]$ & 0.35 & $\mathrm{~m} / \mathrm{m}$ \\
\hline Asperitiy Radius of Curvature $[R]$ & 1 & $\mu \mathrm{m}$ \\
\hline Yield Stress $\left[\sigma_{y s}\right]$ & 136 & $\mathrm{MPa}$ \\
\hline Fracture Initial Height $\left[H_{o}\right]$ & 1 & $\mathrm{~mm}$ \\
\hline Asperity Initial Height $\left[h_{o}\right]$ & 9.86 & $\mu \mathrm{m}$ \\
\hline
\end{tabular}

$a^{2}=\frac{F}{\pi \kappa}$,

where $\kappa$ is the hardness of the material ( $\kappa$ is about three time the yield stress of the material [5]). From Equations (4) and (8) it follows that

$\alpha_{p}=\frac{P-P_{\text {critical }}}{2 R \pi \kappa n}$,

where $P_{\text {critical }}$ is the critical pressure when yielding initiates. According to Etsion et al. [7], the critical deformation at the onset of yielding is given by

$\alpha_{c}=\left(\frac{\pi C_{p} H}{2 K}\right)^{2} R$

where $C_{p}$ is the hardness coefficient given by

$C_{p}=0.454+0.41 v$.

Equation (10) indicates when plastic deformation occurs. This entails the use of Equation (8) to compute the asperity deformation. The effective fracture aperture, $h$, is given as

$h=h_{o}-\alpha$,

where $h_{o}$ is the aperture size at the initial state, and $\alpha$ is given by

$\alpha=\left\{\begin{array}{l}\alpha_{e} \text { for } \alpha<\alpha_{c} \\ \alpha_{\mathrm{e}}+\alpha_{p} \text { for } \alpha \geq \alpha_{\mathrm{c}}\end{array}\right\}$.

The corresponding permeability is then given by Equation (3), where $h$ is given by Equation (12). Table 1 presents the parameters used for modeling the idealized fracture. These parameters were obtained from Hobbs [11]; he conducted experiments to determine the strength and stress-strain characteristics of coal in triaxial compression.

\subsection{Hysteretic Model}

In this section, we outline an alternate method to model the hysteresis in the coal permeability results of Smith et al. [3]. The Bock-Wen model for hysteretic materials was developed by Wen et al., [12]. The thermodynamical basis of the model was studied by Ahmadi et al., [13]. The model consists of a spring, $\lambda$, a viscous damper, $\beta$, and a hysteretic element, $z$, shown in Fig. (3).

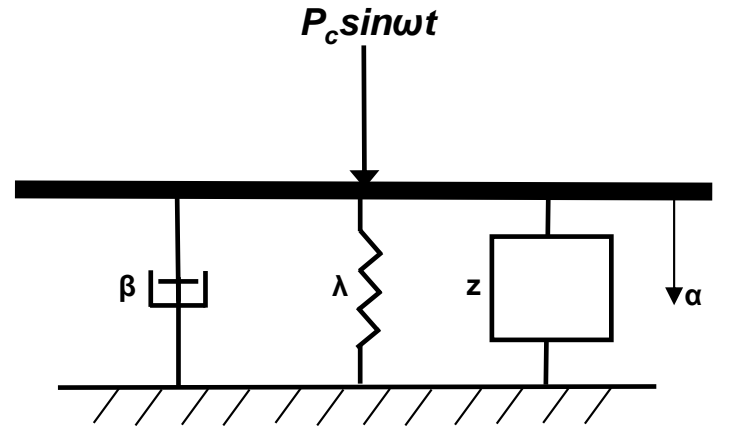

Fig. (3). Schematic of the idealized model for hysteretic behavior of the fracture.

The governing equations of the force-displacement relation are given as

$$
F_{B-W}=\beta \dot{\alpha}+\lambda \alpha+\varepsilon\left(g-b|z|^{n}\right) z
$$

and

$\dot{z}=-\left.u|\dot{\alpha}| z\right|^{n-1} z+\left(g-b|z|^{n}\right) z$

where $F_{B-W}$ is the Bock-Wen hysteretic force, $\alpha$ is the deflection, $n$ is the exponent controlling the sharpness of yielding, and $g, b, u$, and $\varepsilon$ are the parameters controlling the shape of the hysteresis loop. The purposes of these parameters are as follows: the width of the hysteresis loop is controlled by $g, b$ controls the " $\mathrm{S}$ " shape of the hysteresis loop, and $u$ and $\varepsilon$ control the "smoothness" of the hysteresis loop.

For an imposed cyclic confining pressure, $P_{c}|\sin \omega t|$, the governing equation of motion is given as [13]

$m \ddot{\alpha}+F_{B-W}=P_{c}|\sin \omega t|$,

where $P_{c}$ is the peak value of confining pressure, $t$ is the time, $m$ is the mass, and $\omega$ is the frequency of the cyclic loading. The material parameters used in the governing equations are shown in Table 2. The prescribed parameters were chosen to produce fracture asperity deformations similar to the experimental results.

\section{RESULTS}

\subsection{Elastic-Plastic Model Predictions}

In this section, the elastic-plastic model predictions for aperture size, elastic and plastic deformation of the asperities, and the permeability of the coal are presented, and 
Table 2. Hysteretic Model Material Parameters

\begin{tabular}{|c|c|c|}
\hline Symbols & Values & Units \\
\hline \hline$\varepsilon$ & 1.1 & $\mathrm{~N} \mu \mathrm{m}$ \\
\hline$g$ & 2.5 & $\mu \mathrm{m}^{-1}$ \\
\hline$b$ & 0.05 & $\mu \mathrm{m}^{-1}$ \\
\hline$u$ & 0.05 & $\mu \mathrm{m}^{-1}$ \\
\hline$n$ & 1.1 & $\mathrm{~N} \mathrm{~s} / \mu \mathrm{m}$ \\
\hline$\beta$ & 0.05 & $\mathrm{~N} / \mu \mathrm{m}$ \\
\hline$m$ & 2 & $\mathrm{~kg}$ \\
\hline
\end{tabular}

the permeability results are compared with the experimental data of Smith and Jikich [3]. The material properties employed in the analysis are for Pentremawr coal (see Table 1). Figs. (5) and (6) show the predicted permeability as a function of confining pressure for loading and unloading scenarios. Permeability decreases with an increase in the confining pressure. The effective permeability of the idealized fracture model for the unloading condition, however, does not recover fully after unloading. That is, for the same confining pressure, the permeability after unloading is lower than that during the loading condition. Moreover, upon unloading, the effective permeability for the idealized fracture model recovers less than the experiments. This observation clearly points to the hysteretic effects in the coal bed permeability. The elastic-plastic model predictions are in qualitative agreement with the experimental data of Smith and Jikich [3] for the range of confining pressure studied in the experiments. For the loading condition, the general trend of variation of the permeability with confining pressure is properly predicted. However, the experimental data exhibit steeper variations with the confining pressure at the beginning of the loading process.

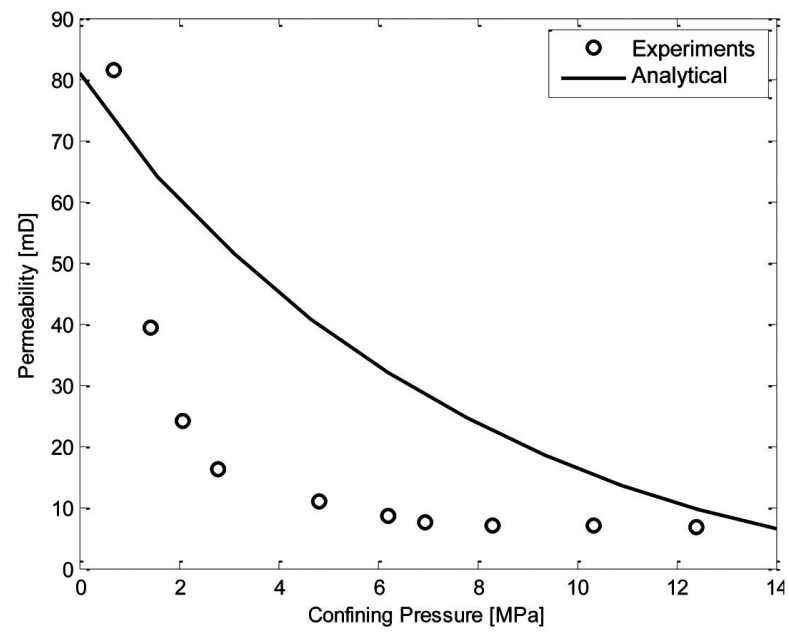

Fig. (4). Variation of permeability with confining pressure for the loading condition for the elastic-plastic model (experimental results from Smith and Jikich [3]).
For the unloading conditions, the elastic-plastic model predictions are in good agreement with the experimental data between 4-14 MPa confining pressures. However, for smaller confining pressures, the predicted permeability is much lower than the experimental values due to residual plastic aperture closure as discussed below.

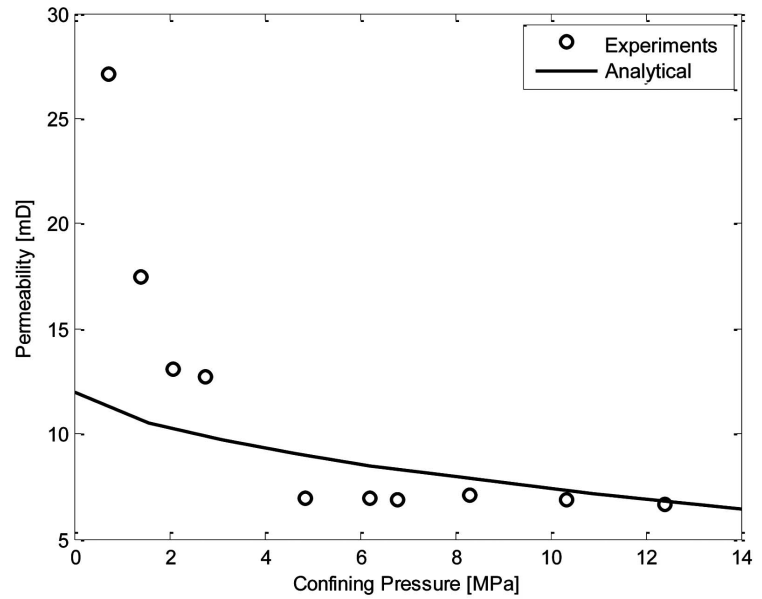

Fig. (5). Variation of permeability with confining pressure for the unloading condition for the elastic-plastic model (experimental results from Smith and Jikich [3]).

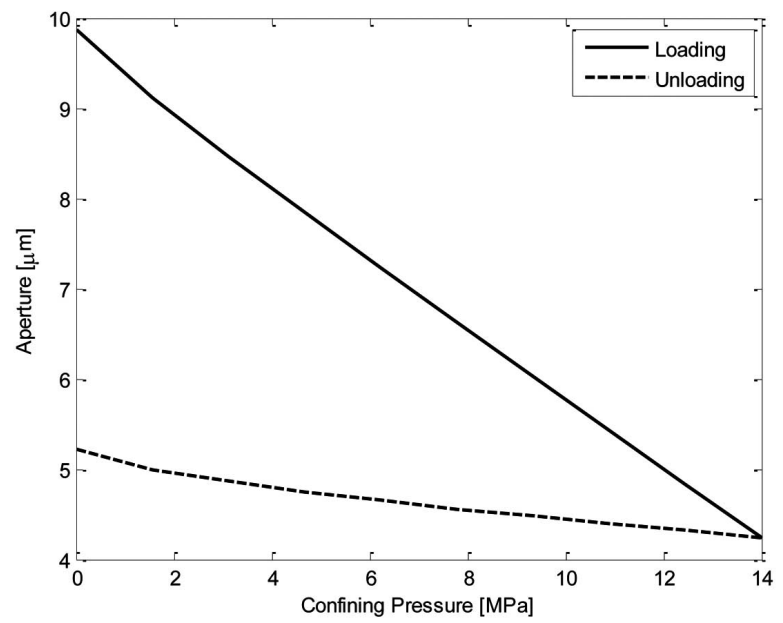

Fig. (6). Variations of predicted fracture aperture with confining pressure for the loading and unloading conditions. 
The experimental results shown in Figs. (4) and (5) suggest that irrecoverable deformation was encountered during the loading and unloading - that is, residual strains were generated in the fracture asperities that caused the fracture aperture to not fully recover and resulted in the reduction of the permeability after a cycle of loading and unloading. In Fig. (6), the predicted fracture aperture is plotted as a function of confining pressure for the loading and unloading scenarios. For the loading conditions, it is seen that the fracture aperture decreases roughly linearly with the confining pressure. For the unloading conditions, the aperture increases at a much lower slope, reaching slightly more than $5 \mu \mathrm{m}$. This is due to the plastic deformation that remains upon unloading. Plastic deformation occurs when stresses in a material body exceed its yield strength. In the present work, the plastic behavior occurs when the asperity penetration exceeds the critical deflection, as seen in Fig. (7). This information can be useful in understanding how fractured surfaces with contacted asperities will deform plastically under overburden pressure.

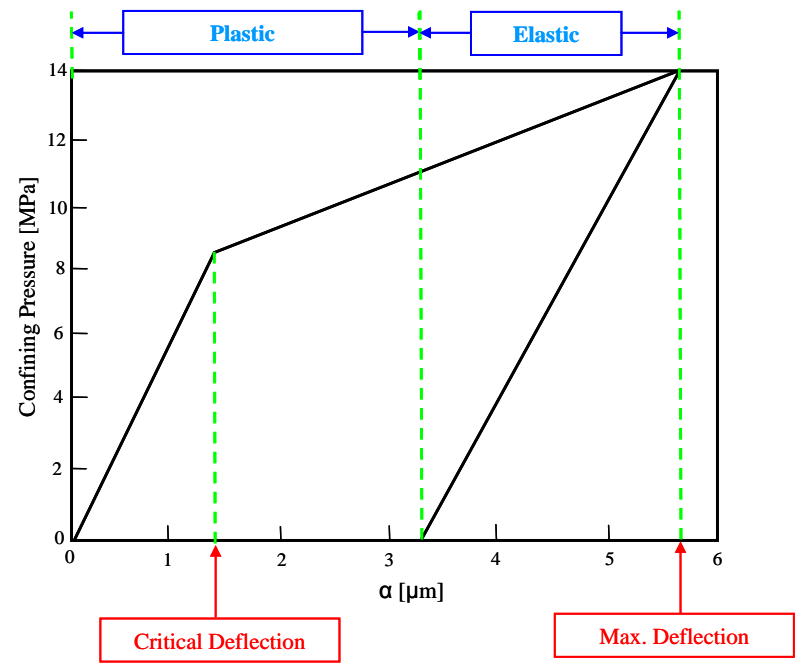

Fig. (7). Variation of combined elastic and plastic deformation of fracture asperities with confining pressure.

In Fig. (7), the total deformation of the asperity is plotted as a function of confining pressure. Fig. (7) shows that yielding of the asperity occurs when the confining pressure is approximately $8.1 \mathrm{MPa}$. At a confining pressure of 14 $\mathrm{MPa}$, the $9.86 \mu \mathrm{m}$ asperity experienced a total deflection of $5.5 \mu \mathrm{m}$. Upon unloading, $2.2 \mu \mathrm{m}$ of elastic deflection was recovered, while $3.3 \mu \mathrm{m}$ of plastic deformation remained. Recalling that the predicted permeability after unloading is less than experiments, a possible cause of this error is that the residual plastic strain from the model is too large. From Equation (9), the plastic deflection can be lowered in three different ways: increasing the critical pressure $\left(P_{\text {Critical }}\right)$, increasing the hardness $(\kappa)$, or increasing the radius of curvature of the asperity $(R)$. Any one or all of these adjustments would in turn provide more permeability recovery upon unloading.

\subsection{Hysteretic Model Predictions}

In this section, the hysteretic model predictions are presented, and the results are compared with the experimental data of Smith and Jikich [3]. The hysteretic model was loaded cyclically under a sinusoidal pressure to obtain three hysteresis loops. This provided a history of deformations of the fracture asperity during loading and unloading. To achieve this, the hysteretic model was run for three cylces. The computed deflections in Fig. (8) were utilized to evaluate the permeability of the fracture, as seen in Fig. (9).

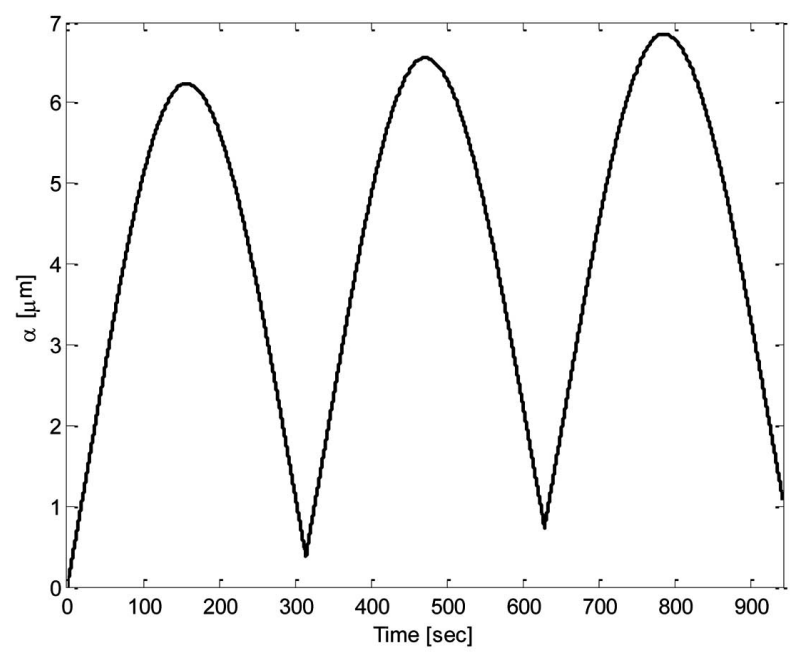

Fig. (8). Predicted displacement of the fracture asperity as a function of time (experimental results from Smith and Jikich [3]).

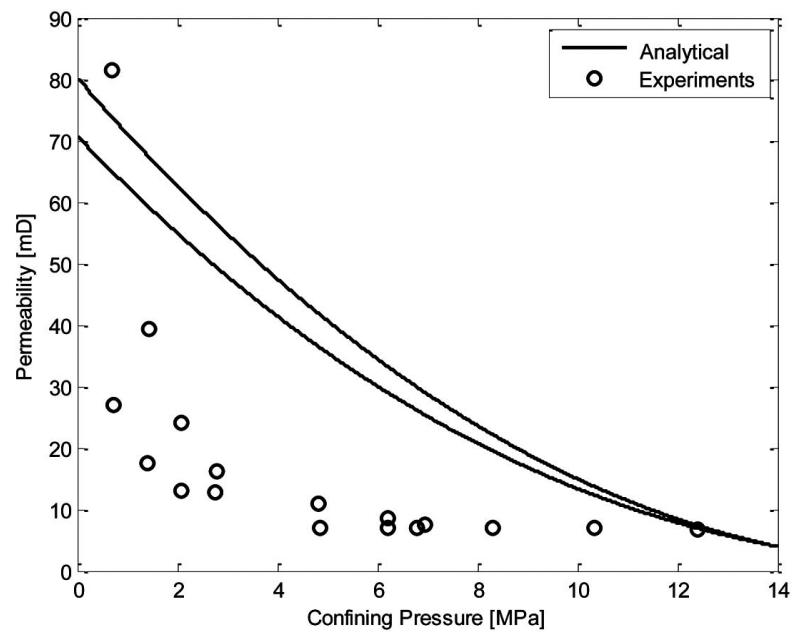

Fig. (9). Variation of permeability with confining pressure of the first cycle for the hysteretic model (experimental results from Smith and Jikich [3]).

Fig. (8) shows the time history of the predicted asperity deflection for a cyclic loading of $\mathrm{P}_{\mathrm{c}}=|\operatorname{sint}|$ with $\mathrm{P}_{\mathrm{c}}=14$ $\mathrm{MPa}$. This figure depicts the progression of permanent deformation of the fracture asperity during the simulation. The residual deflection for cycles 1,2 , and 3 were found to be $0.432 \mu \mathrm{m}, 0.798 \mu \mathrm{m}$, and $1.23 \mu \mathrm{m}$, respectively. Furthermore, the maximum deflection for cycles 1, 2, and 3 were found to be $6.34 \mu \mathrm{m}, 6.57 \mu \mathrm{m}$, and $6.83 \mu \mathrm{m}$, respectively.

The evolution of the hysteresis is apparent in the first cycle, and becomes more severe--that is, less deformation is recovered, and more plastic deformation is accumulated for subsequent cycles. 
The predicted fracture permeability as a function of the cyclic confining pressure is shown in Fig. (9) for the first cycle. Fig. (9) also illustrates the predicted and the experimental permeability for the first cycle. The hysteretic model captured a similar trend as the experiments. However, there are discrepancies between the experiments and the hysteretic model results. The hysteretic model experienced only a reduction in permeability of approximately $10 \mathrm{mD}$ during loading and unloading, while the reduction in the experiments was approximately $50 \mathrm{mD}$. Additionally, the hysteretic model does not exhibit the rapid drop in the permeability under loading, as seen in the experiments. During loading, reasonable agreement was observed between the permeability results of the hysteretic model and the elastic-plastic model. Unlike the hysteretic model, the elastic-plastic model experienced a substantial reduction in permeability when fully unloaded. Thus, the elastic-plastic model seems to better reproduce the experimental results.

\section{DISCUSSION}

The discrepancies between the experiments and the theoretical models are likely a result of the modeling assumptions implemented. First, the parallel-plate assumption is an idealization because a real fracture surface is rough and tortuous. Second, due to variations in fracture aperture in a real fracture, the flow through the fracture moves along a preferred path, so the constant width between the parallel plates is also an idealization. Third, the friction associated with the rough fracture affects the flow performance. Surface roughness in fluid flow through fractured media can result in the transition from laminar flow to turbulent flow. Furthermore, it can affect the pressure gradient in the flow field. Finally, a more realistic plasticity model could be used. Geo-materials are frictional materials that deform plastically by thermodynamically irrecoverable processes. A better correlation might be achieved if a pressure-dependent constitutive model was implemented for the fracture.

\section{CONCLUSIONS}

A simple, elastic-plastic, mathematical model was developed and implemented to determine permeability of fluid flow through fractured media under various levels of confining pressure. The model consisted of a representative element containing a single fracture. Alternatively, hysteresis loops, via the Bouc-Wen model [13], were also used to estimate changes in permeability.

The models for evaluating permeability of fractured media were applied to Pentremawr coal [9] samples. The results obtained from the elastic-plastic and hysteresis models produced similar trends as the experimental data for the range of confining pressures used in the experiments. The elastic-plastic model was better able to simulate residual permeability loss upon unloading. However, notable differences remain between the models and experiments, particularly for the unloading, where residual permeability was either under or over estimated by the models. Model refinements, as described above, could lead to improved results. The model could be incorporated into the available commercial reservoir engineering codes that can be used for providing engineering estimates of the amounts of $\mathrm{CO}_{2}$ that can be sequestered. Such information could be utilized to perform optimizations of carbon sequestration process, by selection of appropriate injection pressures and flow rates. In addition, the model may find applications in fracturing of shale reservoirs for natural gas production.

\section{CONFLICT OF INTEREST}

\section{Declared none.}

\section{ACKNOWLEDGEMENT}

The support of the Department of Mechanical and Aeronautical Engineering at Clarkson University throughout this project is gratefully acknowledged.

\section{REFERENCES}

[1] J.B. Walsh, "Effect of pore pressure and confining pressure on fracture permeability", Int. J. Rock Mech. Min. Sci. Geomech., vol. 18, pp. 429-435, 2003.

[2] J.B. Walsh, and M.A. Grosebaugh, "A new model for analyzing the effect of fractures on compressibility", J. Geophys. Res., vol. 84, pp. 3532-3536, 1979.

[3] D.H. Smith, and S.A Jikich, Effects of fluid, confining, and effective pressure on the helium permeability of upper freeport bituminous coal: measurements and interpretation, National Energy Technology Laboratory, U.S. Department of Energy, 2009.

[4] Y.F. Gao, A.F. Bower, K.S. Kim, L.C. Lev, and Y.T. Cheng, "The behavior of an elastic-perfectly plastic sinusoidal surface under contact loading", Wear, vol. 261, pp. 145-154, 2006.

[5] V. Krithivasan, and R. Jackson, "An analysis of three-dimensional elasto-plastic sinusoidal contact", Tribol. Lett., vol. 27, pp. 31-43, 2007.

[6] Y. Kadin, Y. Kligerman, and I. Etsion, "Unloading an elasticplastic contact of rough surfaces", J. Mech. Phys. Solids, vol. 54, pp. 2652-2674, 2006.

[7] I. Etsion, Y. Kligerman, and Y. Kadin, "Unloading of an elasticplastic loaded spherical contact", Int. J. Solids Struct., vol. 42, pp. 3716-3729, 2005.

[8] R. Jackson, I. Chusoipin, and I. Green, "A finite element study of the residual stress and deformation in hemispherical contacts", $J$. Tribol., vol. 127, pp. 484-493, 2005.

[9] K.L. Johnson, Contact Mechanics, Cambridge University Press: Cambridge 1985, pp. 84-104.

[10] D.Maugis, and H.M Pollock, "Surface forces, deformation and adherence at metal microcontacts", Acta Metallurg., vol. 32, pp. 1323-1334, 2003.

[11] D.W. Hobbs, "The strength and the stress-strain characteristics of coal in triaxial compression", J. Geol., vol. 72, pp. 214-231, 1962.

[12] Y.K. Wen, "Method of random vibration of hysteretic system", $J$. Eng. Mech., vol. 102, pp. 249-263, 1976.

[13] G. Ahmadi, F.C. Fan, and M. Noori, "A thermodynamically consistent model for hysteretic material", Iran J. Sci. Technol., vol. 21, pp. 258-278, 1997 